\title{
Are Drug-Resistant and Drug-Sensitive Patients the Same?
}

\author{
Martin J Brodie \\ Clinical and Research Director, Epilepsy Unit, Western Infirmary, Glasgow, Scotland, UK
}

Key words: antiepileptic drugs, epilepsy, prognosis, seizures

Published online April 11, 2010

\section{Summary}

Around $30 \%$ of adults with epilepsy remain refractory to antiepileptic drug (AED) therapy despite having similar seizure semiologies and syndromes to drug responsive individuals. How, when and why do they become pharmacoresistant? Observations from the Glasgow database of newly diagnosed patients receiving their first ever AED at the Epilepsy Unit have provided fascinating insights into the natural history of treated epilepsy. Prospective analyses of this expanding cohort were undertaken in $1997(\mathrm{n}=470), 2003(\mathrm{n}=780)$ and, most recently, in 2008 ( $n=1098)$. Overall, around 50\% of patients became seizure free on their first ever AED with diminishing numbers responding to subsequent regimens either as mono- therapy or in low dose combinations. The overall prognosis has modestly improved over recent years as newer drugs with novel mechanisms of action have become available. However, from the most recent analysis, around $25 \%$ of the population never had useful period of seizure freedom despite receiving many AEDS singly and in combination. Interestingly, a similar number never had another seizure after starting treatment. Differences between these clinical phenotypes included higher seizure densities prior to initiation of therapy and concurrent psychiatric comorbidities suggesting greater brain dysfunction. The presence of a family history of epilepsy and/or febrile convulsions also predicted refractory 
epilepsy, implicating a genetic component in drug response. These observations tend to support the suggestion that drug-resistant and drug-sensitive patients are not necessarily the same.

\section{Introduction}

Around $5-10 \%$ of people will have a seizure at some time in their lives, $30 \%$ of whom will go on later to develop epilepsy. Thus $1 \%$ of the world's population will have epilepsy at any given point in time, amounting to a total of around 40 million people. Of these, around $30 \%$ of adults remain refractory to pharmacotherapy despite the availability of an increasingly wide range of established and newer drugs (1-4). Uncontrolled epilepsy can be associated with cognitive deterioration, psychosocial dysfunction and increased morbidity and mortality (5-6). Refractory epilepsy represents a socioeconomic burden at the individual, family, societal and political level in all countries across the globe (7). How, when and why do people with epilepsy become pharmacoresistant?

\section{Background}

The Epilepsy Unit at the Western Infirmary, Glasgow, in Scotland, was set up in 1982. From the outset all referred patients, including those with newly diagnosed epilepsy, were entered prospectively into a database. The majority of untreated patients came by direct referral from local general practitioners. From 1990 onwards, following a survey of patients reviewed at the Accident and Emergency Department, all individuals pre- senting there with untreated seizures were reviewed rapidly at the first seizure clinic (8). These experiencing a single seizure were given an emergency number to call should they have another event. This programme has allowed the gradual accumulation of more than 1000 patients, who were diagnosed and started on their first ever antiepileptic drug (AED) under our supervision. This database continues to provide a number of important insights into the natural history of treated epilepsy in adolescents and adults.

\section{Patients}

Most patients with first seizures or untreated epilepsy were reviewed within two weeks of referral, often a day or two after receipt of a faxed letter or telephone call. The current policy is to see urgent cases daily at the Epilepsy Unit rather than allow a waiting time to develop at the clinic. Data are collected manually via a structured questionnaire and later inserted into an electronic database. Routine investigations, including electroencephalography and brain imaging, are undertaken with minimal delay. Classification of seizure types and epilepsy syndromes takes place throughout the follow-up process. For publication purposes, each patient is reviewed again at the time of analysis when as much clinical information as possible has been accumulated.

Monotherapy was employed initially in all patients. Treatment schedules were modified as necessary based on clinical response and drug tolerability. Patients developing idiosyncratic reactions, such as rash, or experiencing 
intolerable side effects, such as sedation, at low AED doses, were deemed to have failed treatment because of adverse effects. Those who continued to experience seizures despite tolerating high doses of medication were designated as treatment failure due to lack of efficacy. Patients not tolerating their first AED were prescribed an alternative. Those failing treatment because of lack of efficacy either had the original drug substituted or were offered combination therapy depending on the clinical status of the patient and their personal preference. The extent of seizure control was assessed at the time of the patient's last clinic visit.

Patients were divided into 5 outcome groups (9). Responders to treatment required to be seizure free for at least 12 months. If they did not report any further seizures, they were regarded as having attained remission. Patients having no further seizures after taking their first AED dose were categorised as immediate responders. Relapse was defined as complete seizure control for at least 12 months followed by the subsequent development of refractory epilepsy. Non- responders to AED therapy never documented any 12 month seizure-free period from the outset.

\section{Outcomes}

The original observations in the first 470 untreated patients with newly diagnosed epilepsy were published in three papers $(3,10$ 11). A subsequent analysis focused on a total of 780 patients with a median age of 29 years (9) followed up between July 1982 and May 2001 [median duration of treatment 6.6 years (range 2-21 years)]. Overall, 59.2\% achieved remission, $53 \%$ of whom were categorised as immediate responders because they had no further seizures after treatment was introduced. Non-responders accounted for $35.4 \%$ of the complete population, whereas $5.4 \%$ (8.4\% of responders) relapsed and never again achieved seizure control.

There were no significant differences in outcome between the cryptogenic (56\% remission) and symptomatic (57\% remission) patient populations, although patients with idiopathic generalised epilepsy syndromes were more likely to become seizure free $(66 \%$ remission). Patients with underlying cortical atrophy (71\% remission; $\mathrm{p}<0.05$ ) or cerebrovascular disease (70\% remission; $\mathrm{p}<0.01$ ) fared better, while those with diffuse brain injury due to trauma (35\% remission; $\mathrm{p}<0.001$ ) did worse than the remainder of the symptomatic group (12). Remission rates in patients with underlying cortical dysplasia (60\%), hippocampal atrophy (50\%) and primary brain tumour (52\%) appeared no different from those with other symptomatic epilepsies. Overall, 20-40\% patients with each epilepsy syndrome reported no further seizures after starting AED treatment including 21\% with hippocampal atrophy and 33\% with cortical dysplasia (12).

Figure 1 compares the prognostic categories from (a) the complete population with those in (b) patients with juvenile myoclonic epilepsy (JME) and (c) those with mesial temporal lobe epilepsy and hippocampal atrophy. Thus, some patients with a potentially benign epilepsy syndrome, ie JME, did not 
(a)

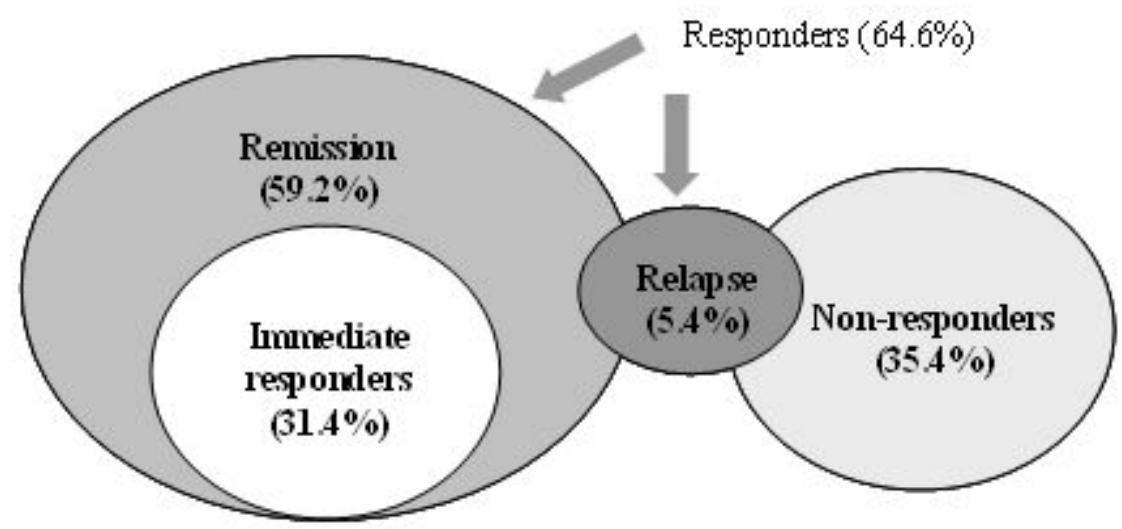

(b)

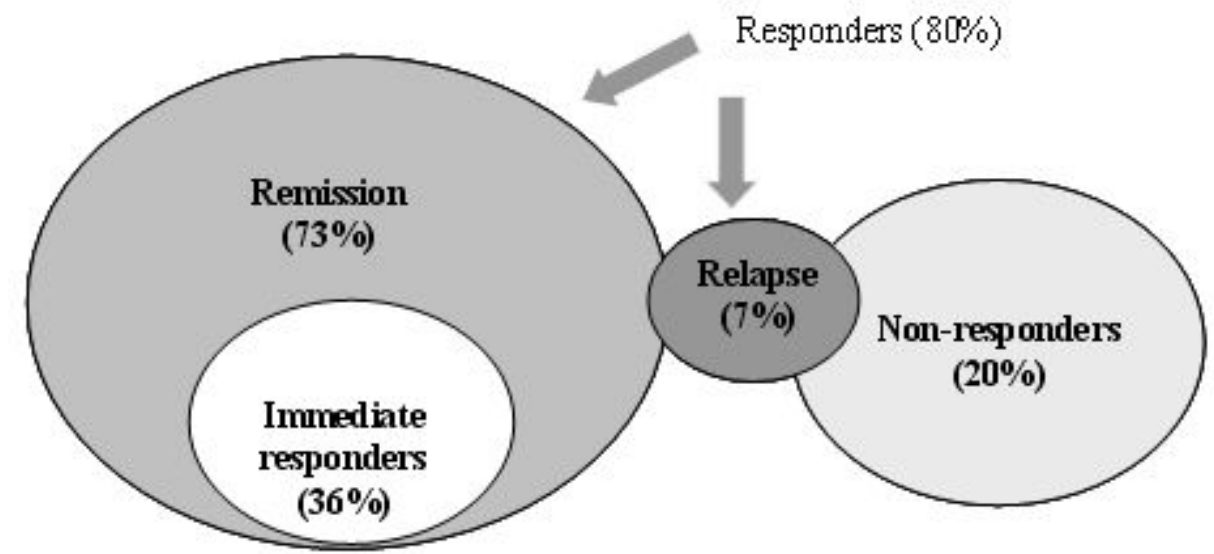

(c)

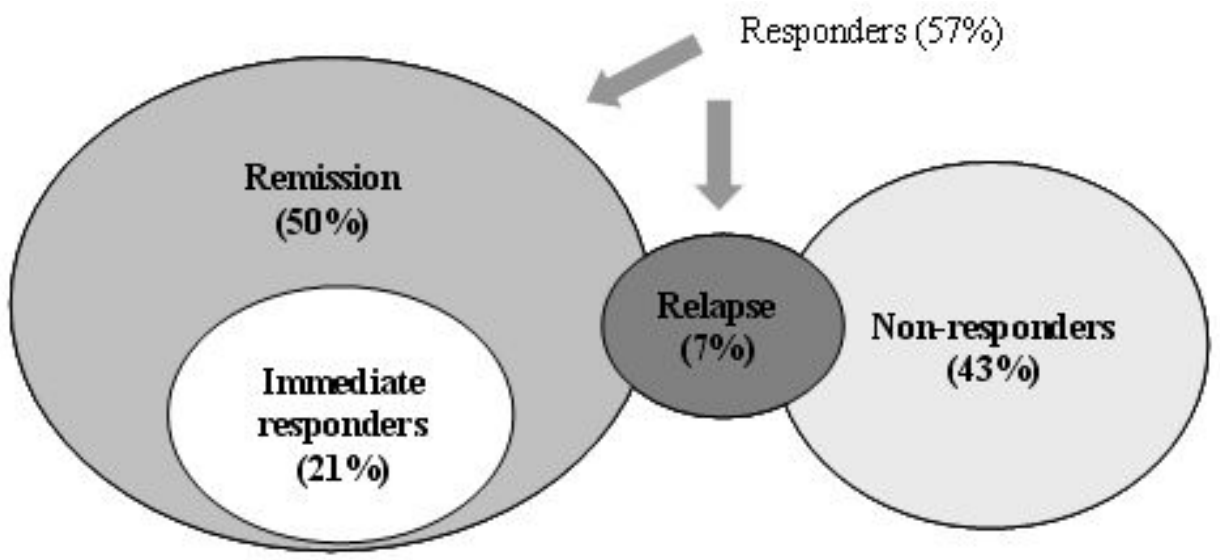

Figure 1. Outcomes in (a) all patients with newly diagnosed epilepsy ( $\mathrm{n}=780)$, (b) those with juvenile myoclonic epilepsies $(n=55)$ and (c) those with mesial temporal lobe epilepsy with hippocampal atrophy $(n=14)$. Data taken from references 9, 12 and 13. Categories in patients with newly diagnosed epilepsies include: responder - seizure-free for a minimum of 12 months; immediate responder - no further seizures after starting treatment; remission - no relapse after responding for at least 1 year; relapse - controlled for at least 1 year and then refractory; nonresponder - never seizure-free for any 12 month period. 
respond to AED therapy (13), whereas other individuals with localization-related epilepsy on a background of hippocampal sclerosis never had another seizure after taking the first AED dose (12). Outcomes in patients with newly diagnosed localization-related epilepsy and concomitant cortical dysplasia also showed a wide variety of drug responses with $60 \%$ attaining remission and 33\% not responding to treatment from the outset (12).

\section{Predictive factors}

A number of studies in adolescents and adults have explored predictors of pharmacoresistant epilepsy among the common seizure types $(1-2,14)$. High numbers of pretreatment seizures have been universally associated with poorer outcomes (15-17). We have recently added to this the observation that high seizure density within a few months of starting AED therapy was a better predictor of subsequent pharmacoresistance than overall pre-treatment seizures numbers (9). The parallel observation that duration of untreated epilepsy did not predict outcome supports the suggestion that responders to AED therapy may represent a different population from non-responders. These observations may help explain the poorer overall prognosis in some active localisation-related epilepsy syndromes, such as those with hippocampal atrophy and cortical dysplasias, where pretreatment seizure frequency and density is often high (18-19). However, as discussed above, some patients with these syndromes have a more benign course (12, 20-21).

Other predictive factors for poor outcome in patients attending our service have included family history of epilepsy, prior febrile convulsions, traumatic brain injury as a cause of the epilepsy, intermittent recreational drug use, and prior or concurrent psychiatric comorbidity, particularly depression (14) Interestingly, Kanner and coworkers reported recently that a lifetime history of depression was the sole predictor of disabling seizures in patients failing temporal lobectomy (22). These two concordant observations from very different patient populations suggest that the deleterious neurobiological processes that underpin depression and perhaps other psychiatric disorders interact with those producing seizures to increase the extent of brain dysfunction and thereby the likelihood of developing pharmacoresistant epilepsy (23). Elderly patients, on the other hand, tended to have a better prognosis (9), with $79 \%$ attaining remission overall, perhaps reflecting lower lesional epileptogenicity and genetic predisposition in this population (24).

\section{Antiepileptic drugs}

Large scale observational studies and randomised controlled trials have demonstrated that around $50 \%$ of people with newly diagnosed epilepsy will control on their first ever AED, often at modest or moderate doses (11, 25-28). The strongly predictive value of response to first AED as a measure of subsequent longterm outcome has been confirmed in other studies (29). Substitution for or combination with the first AED produced seizure freedom in $32 \%$ and $27 \%$ of our patients (25). The remission rate after failing a first drug due 
to adverse effects (42\%) was better than that following failure due to lack of efficacy (21\%). Fewer patients with either idiopathic or localization-related epilepsies did well, however, after failing 2 or 3 drugs schedules for any reason (9).

In the 780 patients starting on treatment between July 1982 and May 2001, 245 (31\%) never had another seizure after taking their first AED dose, whereas a further 276 (35.4\%) never became seizure-free for any 12 month period (Figure 1). Median daily doses of carbamazepine, sodium valproate and lamotrigine in the immediate responders were 469mg, 890mg and 143mg, respectively, suggesting substantial pharmacosensitivity in these individuals (9). Patients with refractory epilepsy were more likely to be male, to have frequent pre-treatment seizures and to have cyptogenic epilepsy (9).

\section{Randomized trials}

Many active-controlled, double-blind and pragmatic randomised trials have been undertaken in patients with newly diagnosed epilepsy with the established and newer AEDs (26-28, 30-31). Comparisons between phenobarbital, primidone, phenytoin, carbamazepine and sodium valproate have shown no difference in efficacy among these drugs, although phenobarbital and primidone in highish doses tended to be less well tolerated (32). Second generation AEDs, such as lamotrigine, gabapentin and oxcarbazepine have demonstrated a tendency toward better tolerability, but again no important differences in efficacy have been reported compared with established
AEDs, suggesting that some patients will respond to any appropriate first choice despite differences in their mechanisms of action (31).

These observations have been augmented by the recently published double-blind, randomised controlled trial comparing controlled-release carbamazepine and levetiracetam in this patient population (28). These drugs have substantial pharmacological differences. Carbamazepine is a traditional narrow spectrum AED that binds to inactivated neuronal $\mathrm{Na}^{+}$channels to produce a voltage and frequency-dependent reduction in conductance. Levetiracetam has efficacy for idiopathic generalised epilepsies as well as for partial seizures (33). It binds to synaptic vesicle 2A protein, thereby interfering with the neuronal release of a range of neurotransmitters. This study was unique in that seizure-free patients could remain on the lowest effective dose of either treatment. The design allowed patients to be followed up until they were seizure-free for a year.

Despite the above pharmacological differences between the comparators, identical outcomes were obtained with both AEDs (28). Interestingly, Kaplan-Meir curves for time to discontinuation due to lack of efficacy or poor tolerability were superimposible using either the per protocol or intention-to-treat analysis. Overall, $56.6 \%$ and $58.4 \%$ of patients randomized to levetiracetam or controlled-release carbamazepine remained seizure-free for one year using the per protocol analysis with $89 \%$ and $86 \%$, respectively, taking the lowest dose (levetiracetam 500mg twice daily or carbamazepine $200 \mathrm{mg}$ twice daily). The overall 
one year seizure free rate in the intention-totreat population was very similar to the figure obtained from the Glasgow population of newly diagnosed patients (Glasgow database 50\%; carbamazepine 53\%; levetiracetam $50 \%)$. Thus, this trial offered confirmation in a randomized, double-blind setting of previous uncontrolled observations that most people with newly diagnosed epilepsy will respond to their first ever AED at low dosage.

\section{Recent observations}

The question "Are drug-sensitive and drug-resistant patients the same?” is an important one. If they are not, it should be possible to identify individuals destined to develop refractory epilepsy early and devise a specific therapeutic strategy for them. Although we now have available more than 20 AEDs with an apparently wide-range of mechanisms of action, all target similar phar- macological endpoints by decreasing neuronal excitation and increasing neuronal inhibition (34). Thus, one might expect the overall prognosis to improve slowly in the refractory epilepsy population as newer AEDs that work in different ways become more generally available. In support of this suggestion is the emerging evidence that some of the newer broad spectrum drugs may be making inroads into the $30-40 \%$ of patients with pharmacoresistant epilepsy (35-36).

Our ongoing analysis supports this observation with preliminary results being compared to previous published data in Table 1. The increase in seizure-free rates with polypharmacy but not monotherapy suggests that newer agents, such as topiramate, levetiracetam and zonisamide, with different mechanisms of action are having a modest impact on overall outcome. Of 1098 patients with newly diagnosed epilepsy in our most

Table 1. Remission rates in an expanding cohort of patients with newly diagnosed epilepsy preparation

\begin{tabular}{|c|c|c|c|c|}
\hline Recruitment & $\mathrm{N}$ & Monotherapy & Polypharmacy & Total \\
\hline $1982-1997^{1}$ & 470 & $61 \%$ & $3.0 \%$ & $64.0 \%$ \\
\hline $1982-2003^{2}$ & 780 & $59 \%$ & $5.4 \%$ & $64.4 \%$ \\
\hline $1982-2008^{3}$ & 1098 & $62 \%$ & $6.4 \%$ & $68.4 \%$ \\
\hline
\end{tabular}

Data from ${ }^{1}$ Kwan P, Brodie MJ, 2000; ${ }^{2}$ Mohanraj R, Brodie MJ, 2006; ${ }^{3}$ Brodie MJ, Bamagous G, Kwan $\mathrm{P}$, in preparation 
recent unpublished analysis, only $6.4 \%(\mathrm{n}=70)$ were controlled on more than one AED with the vast majority $(96 \%)$ of these $(n=67)$ receiving two AEDs. Because of the broader range of pharmacological options, a number of patients have now been controlled with their $3^{\text {rd }}, 4^{\text {th }}$ and $5^{\text {th }}$ regimens either as monotherapy or with a combination of AEDs. Similar observations have been made by Schiller and Najjar (37). These data provide substantial encouragement for further AED development (34).

The above data raised the question as to whether all patients currently refractory to AED therapy could be controlled with a pharmacological moiety that addresses the neurobiology of their specific epilepsy. A knowledge of the process responsible for seizure development and propagation in the individual person with epilepsy is sadly lacking. Alternative explanations for the development of refractory epilepsy include variations in expression of efflux transporter proteins, alterations in ion channels and receptors and genetically determined factors affecting drug response (38). Lastly, it is possible too that the production of selective brain autoantibodies may result in autoimmune refractory epilepsy in a few individuals (39). The unravelling of these intriguing hypotheses will identify new ways to prevent and treat pharmacoresistance and thereby improve the quality of life of many more people with epilepsy.

\section{References}

[1] Annegers J, Hauser WA, Elverback LR. Remission of seizures and relapse in patients with epilepsy. Epilepsia 1979; 20: 729-737

[2] Cockerell OC, Johnson AL, Sander JWAS, Hart YM, Shorvon SD. Remission of epilepsy: results from the national general practice study of epilepsy. Lancet 1995; 346: 140144

[3] Kwan P, Brodie MJ. Early identification of refractory epilepsy. N Engl J Med 2000; 342: 314-319

[4] Fosgren L, Beghi E, Oun A, Sillanpää M. The epidemiology of epilepsy in Europe - a systematic review. Eur J Neurol 2005; 12: 245-253

[5] Devinsky O. Patients with refractory seizures. New England Journal of Medicine 1999; 340: 1565-1570

[6] Kwan P, Brodie MJ. Refractory epilepsy: a progressive, intractable but preventable condition? Seizure 2002; 11: 77-84

[7] Pugliatti M, Beghi E, Forsgren L, Ekman M, Sobocki P. Estimating the cost of epilepsy in Europe: a review with economic modelling. Epilepsia 2007; 48: 2224-2233

[8] McKee PJW, Wilson EA, Dawson JA, Larkin JG, Brodie MJ. Managing seizures in the casualty department. Brit Med J 1990; 300: 976-979

[9] Mohanraj R, Brodie MJ. Diagnosing refractory epilepsy: response to sequential treatment schedules. Eur J Neurol 2006; 13: 277282

[10] Kwan P, Brodie MJ. Epilepsy after the first drug fails: substitution or add-on? Seizure 2000; 9: 464-468

[11] Kwan P, Brodie MJ. Effectiveness of first antiepileptic drug. Epilepsia 2001; 42: 125560

[12] Mohanraj R, Brodie MJ. Outcomes in newly diagnosed localisation-related epilepsies. Seizure 2005; 14: 318-323

[13] Mohanraj R, Brodie MJ. Outcomes of newly 
diagnosed idiopathic generalised epilepsy syndromes in a non-pediatric setting. Acta Neurol Scand 2007; 115: 204-208

[14] Hititris N, Mohanraj R, Norrie J, Sills GJ, Brodie MJ. Predictors of pharmacoresistant epilepsy. Epilepsy Res 2007; 75: 192-196

[15] Collaborative Group for the Study of Epilepsy. Progress of epilepsy in newly referred patients: a multicenter prospective study of the effects of monotherapy on the long-term course of epilepsy. Epilepsia 1992; 33: 45-51

[16] Sillanpää M. Remission of seizures and predictions of intractability in long-term followup. Epilepsia 1993; 34: 930-936

[17] Leschziner G, Jorgensen AL, Andrew T, Pirmohamed M, Williamson PR, Marson AG, et al. Clinical factors and ABCBI polymorphisms in prediction of antiepileptic drug response: a prospective cohort study. Lancet Neurol 2006; 5: 668-676

[18] Semah F, Picot M-C, Adam C, Broglin D, Arzimanoglou A, Bazin B, et al. Is the underlying cause of epilepsy a major prognostic factor for recurrence? Neurology 1998; 51: 1256-1262

[19] Stephen LJ, Kwan P, Brodie MJ. Does the cause of localisation-related epilepsy influence the response to antiepileptic drug treatment? Epilepsia 2001; 42: 357-362

[20] Andrade-Valenca LP, Valenca MM, Ribeiro LT, Matos ALM, Sales LV, Valesco TR, Santos AC, Leite JP. Clinical and neuroimaging features of good and poor seizure control with mesial temporal lobe epilepsy and hippocampal atrophy. Epilepsia 2003; 55: 807-814

[21] Kobayashi IE, D’Agostino MD, LopesCendes I, Berkovic SF, Andermann E, Andermann F, Cendes F. Hippocampal atrophy and T2-weighted signal changes in familial mesial temporal lobe epilepsy. Neurology
2003; 60: 405-409

[22] Kanner AM, Byrne R, Chicharro A, Wuu J, Frey M. A lifetime psychiatric history predicts a worse seizure outcome following temporal lobectomy. Neurology 2009; 72: 793-799

[23] Jobe PC. Common pathogenic mechanisms between depression and epilepsy: an experimental perspective. Epilepsy Behav 2003; 4: S14-S24

[24] Stephen LJ, Kelly K, Mohanraj R, Brodie MJ. Pharmacological outcomes in older people with newly diagnosed epilepsy. Epilepsy Behav 2006: 8: 434-437

[25] Mohanraj R, Brodie MJ Pharmacological outcomes in newly diagnosed epilepsy Epilepsy Behav 2005; 6: 382-387

[26] Marson AG, Al-Kharusi A, Alwaidh M et al. The SANAD study of effectiveness of carbamazepine, gabapentin, lamotrigine, oxcarbazepine or topiramate for treatment of partial epilepsy: an unblinded randomised controlled trial. Lancet 2007; 369: 1000-1015

[27] Marson AG, Al-Kharusi A, Alwaidh M et al. The SANAD study of effectiveness of valproate, lamotrigine and topiramate for generalised and unclassifiable epilepsy: an unblinded randomised controlled trial. Lancet 2007; 367: 1016-1026

[28] Brodie MJ, Perucca E, Ryvlin P, BenMenachem E, Meenche H-J for the Levetiracetam Monotherapy Study Group. Comparison of levetiracetam and controlledrelease carbamazepine in newly diagnosed epilepsy. Neurology 2007; 68: 402-408

[29] Dlugos DJ, Sammel MD, Strom BL, Farrar JT. Response to first drug trial predicts outcome in childhood temporal lobe epilepsy. Neurology 2001; 57: 2259-2264

[30] Mohanraj R, Brodie MJ. Measuring the efficacy of antiepileptic drugs. Seizure 2003; 12 : 
413-443

[31] Kwan P, Brodie MJ. Clinical trials of antiepileptic medications in newly diagnosed patients with epilepsy. Neurology 2003; 60: (suppl 4): S2-S12

[32] Mattson RH, Cramer JA, Collins JF, Smith DB, Delgado-Escueta AV et al. Comparison of carbamazepine, phenobarbital, phenytoin and primidone in partial and secondary tonicclonic seizures. N Engl J Med 1985; 313 : 145-151

[33] Berkovic SF, Knowlton RC, Leroy RF, Schiemann J, Falter $U$ on behalf of the Levetiracetam No 1057 Study Group. Placebo-controlled study of levetiracetam in idiopathic generalised epilepsy. Neurology 2007; 69: 1751-1760

[34] Kwan P, Brodie MJ. Emerging drugs for epilepsy. Expert Opinion Emerging Drugs 2007; 12: 407-422

[35] Luciano AL, Shorvon SD. Results of treatment changes in patients with apparently drug-resistant chronic epilepsy. Ann Neurol 2007; 62: 375-381

[36] Callaghan BC, Anand K, Hesdorffer D, Hauser WA, French JA. Likelihood of seizure remission in adult population with refractory epilepsy. Ann Neurol 2007; 62: 382-389

[37] Schiller Y, Najjar Y. Quantifying response to antiepileptic drugs: effect of post treatment history. Neurology 2008; 70: 54-65

[38] Kwan P, Brodie MJ. Refractory epilepsy: mechanisms and solutions. Expert Rev Neurotherapeutics 2006; 6: 397-406

[39] Vincent A, Lang B, Kleopa AK. Autoimmune channelopathies and related neurological disorders. Neuron 2006; 52: 123-138 